\title{
INFERENCE OF THE HARD X-RAY SOURCE DIMENSIONS IN THE 1972, AUGUST 7 WHITE LIGHT FLARE
}

\author{
DA VID M. RUST \\ Sacramento Peak Observatory, Air Force Cambridge Research Laboratories, Sunspot, N.M., U.S.A.
}

\begin{abstract}
Summary*. Broadband photographs and spectra of the white light flare of 1972, August 7 have been compared with hard X-ray spectra from the same event. There is a very close temporal correspondence between the hard X-ray and white light emission curves, and these emissions come from layers that are separated by a height of less than $2000 \mathrm{~km}$. The flare shows at least two distinct particle acceleration phases: the first, occurring at a stationary source, gave very bluish continuum emission from 4 bright stationary knots while the X-ray $(E>60 \mathrm{keV})$ spectrum hardened and reached peak intensity. This phase occurred between 1520 and 1523 UT. In the second phase (1524-1537 UT) the bright knots dissolved and a faint wave moved out from the flare center at $40 \mathrm{~km} \mathrm{~s}^{-1}$. The spectrum of the wave was nearly flat in the range $4950-5900 \AA$ and analysis of the spectrum indicates that the emission was probably due to heating and ionization by $20-100 \mathrm{keV}$ electrons. The X-ray spectrum, as derived from Interkosmos 7 and ESRO TD-1A satellite data, becomes softer during the wave phase. The close correspondence between the $\mathrm{X}$-ray and continuum emission events shows that, in effect, the hard X-ray source has been resolved. It consists of several changing patches approximately $3^{\prime \prime} \times 5^{\prime \prime}$ in area, consistent with the upper limit of $1^{\prime}$ from balloon observations (Takakura et al., 1971).
\end{abstract}

\section{References}

Takakura, T., Ohki, K., Shibuya, N., Fuji, M., Matsuoka, M., Miyamoto, S., Nishimura, J., Oda, M., Ogawara, Y., and Ota, S.: 1971, Solar Phys. 16, 454.

* For the full text of the paper see Solar Phys. 40 (1975), 141. 